\title{
ANÁLISIS DEL DISCURSO MEDIÁTICO SOBRE EL SISTEMA EDUCATIVO PÚBLICO'. Análisis del diario La Nación. Mayo de 2007-abril de 2008
}

\author{
ANALYSIS OF MEDIATIC DISCOURSE ABOUT \\ THE EDUCATIONAL PUBLIC SYSTEM. \\ Analysis of La Nación newspaper. May 2007-April 2008
}

\section{Pablo Carballo Chaves*}

\begin{abstract}
RESUMEN
Este artículo brinda una reflexión discursiva sobre la forma en que se trata al sistema educativo público en el diario costarricense La Nación. La visión de discurso se postula desde la metodología del framing, que consiste en la reconstrucción discursiva de marcos de comprensión a partir de los insumos que el diario suministra por medio de sus noticias, principalmente. La reconstrucción del sistema educativo se da a partir de dividir dicho sistema en protagonistas: profesores y estudiantes, junto con el Ministerio de Educación Pública como la institución coordinadora. Así, dentro de lo relevante está atender de nuevo a la tecnología y el inglés, como bases de un modelo de competitividad que la educación debe promover a nivel público, dejando de lado otros soportes fundamentales en la vida de las personas. Además de condicionar, en cierto sentido, el ver a la educación pública como asistencial, más que como derecho $y$ responsabilidad estatal $y$ colectiva.
\end{abstract}

PALABRAS CLAVE: COSTA RICA * LA NACIÓN * ANÁLISIS DE DISCURSO * FRAMING * SISTEMA DE ENSEÑANZA * EDUCACIÓN PÚBLICA * MEDIOS DE COMUNICACIÓN

\section{ABSTRACT}

This article provides a discourse reflection on how La Nación - a Costa Rican newspaper - refers to the educational public system. The view of discourse in this research is centralizing on the framing methodology, which of the reconstruction of discourses which are explained from a comprehension frames based on empirical

$1 \quad$ Este artículo se basa en la investigación de tesis de licenciatura (Carballo, 2009). Asimismo, esta investigación se dio gracias a la importante colaboración del Sistema de Becas de Investigación que facilita el Instituto de Investigaciones Sociales (IIS) de la Universidad de Costa Rica.
Cursando el Posgrado Centroamericano en Ciencias Sociales, FLACSO Costa Rica-Guatemala. carballopablo@gmail.com 
data from the news, mainly. The public education system reconstruction was based in the system divided into actors: teachers and students, together with the national education bureau, Ministerio de Educación Pública, main institution of the area. The most relevant points are: the importance of the technology and English, as a foreign language has in the newspaper, and as reflecting a competitive model which the educational system should be promoted in a public level; leaving aside other elements that are mainstays in the life of people. This way of thinking, presents the public education as an aid system. The perspective of rights, state and collective responsibilities has been diminished in this discourse proposal.

KEYWORDS: COSTA RICA * LA NACIÓN * ANALYSIS OF DISCOURSE * FRAMING * EDUCATIONAL SYSTEM * PUBLIC EDUCATION * MASS-MEDIA

\section{INTRODUCCIÓN}

El campo de estudio de los discursos puede versar sobre diferentes acciones humanas, que están entretejidas en gamas complejas de relaciones sociales. En este sentido, los discursos pueden concentrarse, como lo hace este artículo, en el estudio de la construcción discursiva que un medio de comunicación específico hace sobre una temática específica. El medio de comunicación masiva seleccionado es el diario costarricense La Nación y la temática específica es la educación pública. Sin adentrarse mucho a caracterizar el Diario, se puede señalar de este lo siguiente: es el segundo diario activo de más años (fundado en 1946), después de La Prensa Libre (fundado en 1889); forma parte del grupo empresarial GRUPO NACIÓN SA, el cual se establece como corporación; además dentro de las investigaciones sobre este medio se pueden encontrar análisis sobre su relación con empresas y poder económico y político, el reforzamiento de estereotipos, así como manejo de temas como la violencia — de diferente tipoasí como, estudios ideológicos con perspectivas contextuales de la región, entre otros (Araya, 2008; Beirute, 2006; Arias y Bermúdez, 2006; Pereira y Ramírez, 2004; Martín, 2004; Valitutti, 1992; Sandoval y Al-Ghassani, 1990; Zeledón, 1987 y Jiménez, 1989).

En lo que concierne a la temática analizada en el medio, la principal justificación redunda en señalar que el sistema de educación pública es importante en la forma en como la sociedad costarricense se ha venido desarrollando, así como los cambios y gestaciones que se vienen dando en los últimos años. Lo importan- te es justificar el tema en términos de señalar que está ubicado en un medio de comunicación particular, así la educación pública se puede ver desde un interés público o personal, así como, privado o mediático, en términos de los intereses empresariales, educativos, nacionales y en un plano más general, ideológicos.

La estructura de este artículo es esquemáticamente metodológica, su redacción es plana en el sentido de reflejar lo mejor posible el trascurso procedimental de la investigación en la que se basa. Se exponen una serie de acápites que tienen una estructura similar a las propuestas de tesis. Una sección de discusión teórica $y$ explicación metodológica breve, un espacio para el contexto de la educación pública y su dimensión estatal y social, una exposición de los datos cuantitativos y lo que la interpretación de estos brinda en el estudio de medios, con un ejemplo específico, así como el análisis de fondo en donde se reconstruye el discurso del medio a partir de la herramienta del framing, finalmente, las conclusiones más significativas del trabajo en el que se basa este artículo.

\section{PERSPECTIVA TEÓRICO-METODOLÓGICA}

La centralidad analítica versa sobre un análisis en el discurso que se genera o construye en el medio acerca del sistema de educación público del país. Teniendo presente que lo que se indaga es o son los discursos, la teoría en la que se fundamenta el estudio tiene varias dimensiones, que hablan entre sí, no de forma necesariamente armónica, aunque sí interpretativamente.

Existen diferentes corrientes del análisis de discurso, así como enfoques metodológicos, 
dependiendo del tema, los actores, los recursos, etc. La propuesta de esta investigación es el uso de la técnica llamada framing, que tiene un referente metodológico, así como teórico. Primero se evidencia el debate teórico, después se profundizará la propuesta metodológica seguida a partir de la discusión.

\subsection{DEBATE TEÓRICO SOBRE EL DISCURSO Y LA IDEOLOGÍA}

Hablar o discutir teóricamente sobre el discurso y la ideología es cada vez más complejo y difícil. Las corrientes se han multiplicado, aunque siempre mantienen algunos ejes sobre los cuales establecer puentes de desarrollo, retroceso o debates que estimulen problemas, tanto para solucionar, como para replantear.

Al hacer un barrido rápido sobre los conceptos, se podría señalar que tienen tradiciones distintas, siendo el término y concepto de ideología más antiguo que el concepto discurso. Donde la ideología se puede vincular con las bases rectoras $y$ valorativas de los individuos, mientras que los discursos son la parte más práctica de la vida social, en donde se entrevén las prácticas sociales y las estructuras de poder, que en términos de la reflexión es lo que precisa analizar. Algunos elementos que utilizan el concepto de ideología, usados en este artículo son: sistema de pensamiento, valores, circunstancias políticas, creencias (Mannheim, 2004; López, 2004 y Ariño, 1997). En el caso del discurso se puede acuerpar un marco referencial señalando el que tiene una lógica jerarquizadora (Van Dijk, 2000; 2003), así como, también:

El discurso viene a ser un espacio de encuentros y luchas (base elemental del concepto de relaciones de poder ${ }^{2}$ en

$2 \quad$ El término relaciones de poder se desarrolla en un momento posterior a la publicación del Orden del discurso y Arqueología del saber. Miguel Morey (Morey en Foucault, 1984) señala que el término de poder en la obra de Foucault es tardía; el poder se ubica en el desarrollo del Método Arqueológico del francés. De allí que se advierta que en esta investigación se condensa mucho del pensamiento foucaultiano, tratando de rescatar la explicación sobre las relaciones en el discurso y el uso del poder.
Foucault) en donde se establecen criterios referentes a la acción, no es solamente apropiación de percepciones, sino es el posicionamiento de acciones. Son estructuras que se crean a partir de la delimitación, del entendimiento de mi espacio, y el espacio del otro, de nuestro espacio, y el de otros. El discurso no son los enunciados, el discurso es la práctica de la estructura establecida según la complejidad, por un lado de los enunciados y por otro de los saberes y el poder que con estos se da (Carballo, 2009:27).

La discusión se basa en la relación que hay entre estos dos conceptos con el objetivo de comprender como se decanta la producción mediática de La Nación en esas líneas de interpretación. Es así como, uno de los puntos explicativos $y$ de debate en términos del fundamento teórico, se expresa de la siguiente manera:

... la base rectora - ideología- establece lo que pasó, lo que pasa y lo que ha de pasar, una linealidad de la acción; mientras que la estructura de las relaciones humanas como fenómeno social, llevada a cabo en parte por las relaciones de poder de Foucault, son dimensionalmente variadas, hay condicionalidad $y$ direccionalidad, pero no determinación absoluta (existen nociones de $\operatorname{azar}^{3}$ y flexibilidad discursiva $\left.{ }^{4}\right)$, es decir es variable, no es ni estática, ni determinable en términos de concreción absoluta, sino en términos de conjugación de relaciones forzadas, dependiendo de las propuestas enunciativas que se ejercen en los discursos (Carballo, 2009: 28-29).

Sin embargo, no se debe olvidar la relación de doble vía que hay entre el discurso y la ideología, una nutriendo a la otra en espacios específicos de la vivencia de las personas.

3 La concepción de azar está presente en: Foucault. El orden del Discurso.

4 La concepción de flexibilidad o acomodos discursivos está presente en: Foucault, M. Arqueología del saber. 2006. 
Alrededor de este complejo escenario donde intervienen los términos no directamente abordados de poder, creencias y política, por mencionar unos cuantos, es fundamental darles el arraigo social. En este sentido, lo que se busca en las noticias del periódico o en las notas informativas, es poder precisar estas estructuras de poder, estas creencias, la forma de señalarlas. Es decir, la noticia se ve como una reconstrucción particular hecha por la alianza no necesariamente armónica, aunque puede serlo, entre el periodista y el medio.

... si bien (...) se parte de la noticia como realidad social construida, esta no es más que una de las realidades que los individuos construimos cotidianamente. Se puede discutir la importancia o relevancia de las distintas realidades socialmente construidas. Pero no hay que caer en la falacia de la unicidad de la realidad (Alsina, 1993: 34).

De modo que, la noticia no es ni neutra, ni inocente en términos de su elaboración, ni en términos de su impacto en quienes la consumimos.

Al hacer énfasis en la noticia como parte de una propuesta discursiva del medio de comunicación analizado, se puede rescatar el valor de la temática del sistema de educación pública de la siguiente manera, según dos planos:

1. Que los medios de comunicación masiva participan de la educación de las personas al ser parte del entramado social en el que se desarrollan.

2. Los mass-media abordan y proponen, según la ideología que presentan en la base cognitiva y estratégica de los dueños de medios, una construcción sociocognitiva sobre lo que es, como actúa $y$ lo que debe ser el sistema educativo público.

Con base en lo anterior:

Tenemos entonces una doble dimensión de la situación educativa, por un lado las acciones mediáticas, sus discursos, que se incorporan en el proceso educacional de las personas, $y$ por otro las acciones mediáticas - discursos - que establecen el tipo de sociedad y el modelo educativo que debe aplicarse a ese modelo de sociedad (Carballo, 2000: 49).

La segunda problematización es sobre lo que se centra la reflexión analítica del texto, pero sin olvidar el primer punto. El tema de la educación y del sistema educativo deviene en un tema social y político. Social por su contenido respecto a la relación con las interacciones sociales y la integración de los individuos en un proceso de desarrollo (cfr. Oconnor, 1971) y político porque es un ámbito de discusión institucional formal y relaciones de poder, en donde múltiples discursos se presentan (cfr. Popkewitz, 1997).

\subsection{PROPUESTA TEÓRICO-METODOLÓGICA}

Las discusiones sobre el discurso y la ideología pueden ser retomadas desde diferentes miradas analíticas. Lo postulado en este artículo, se fundamenta en la reflexión metodológico/técnica del framing. Esta técnica, que puede ser retomada tanto teórico-metodológicamente, como centralizada en forma de técnica, postula una visión que redunda en la construcción o reconstrucción de encuadres o lo que otros denominan como esquemas. Un primer acercamiento al enmarcando o framing indica que dicha teoría/metodología consiste en: "Los esfuerzos estratégicos conscientes realizados por grupos de personas para construir interpretaciones compartidas del mundo y de sí mismos que legitiman y motivan la acción colectividad" (Sádaba en Trigueros, 2008: 38). Es así como se da con la relación que tiene una postura del framing incluyendo creencias, interpretaciones, discursos, etc. Esto motiva a pensar en la herramienta, tanto en el nivel de la propuesta teórica, como en la técnica, que tiene un asidero analítico importante.

Según Gitlin (Sádaba, 2004) los frames se vuelven herramientas de elites de diversa índole que los utilizan para orientar la conciencia ciudadana: 
... los frames consiguen que esta elite considera relevante parezca lo natural ante el resto de los ciudadanos (p. 69). Gamson (p.69) plantea, al contrario de Gitlin, que los medios no son los únicos proveedores de frames. Desde distintos orígenes se presentan discursos que con sus múltiples frames construyen mapas que indican puntos relevantes de entrada, salida, atajos y muestran las amenazas del camino. (...) Gamson sugiere que hay que escuchar la voz propia, pero también la voz de los medios y la voz de la cultura que provee un contexto (Trigueros, 2008: 39-40).

La intencionalidad analítica del texto es conformar una discusión acerca del discurso que se presenta en el diario La Nación, a partir de establecer una serie de herramientas de codificación que busquen conformar frames o marcos para la comprensión del fenómeno del sistema educativo público.

Aquí diferentes recursos son fundamentales para que un marco o frame sea asimilable, o en cierta medida retomado para entender $y$ desarrollar una forma de valorar un hecho, una acción, etc. $\mathrm{El}$ frame (marco) viene a ser una herramienta mediática (si se usa según un escenario de interés y relación de poder), la cual se compone a su vez de metáforas, uso de fuentes, calificativos, entre otras, que en su significancia hacen referencia o están conectadas con aspectos simbólicos (lingüísticos o extra-lingüísticos), lo cual da fortaleza de sentido a su existencia y reproducción (Carballo, 2009: 58).

Sin profundizar más en la visión crítica de la técnica, se puede aproximar más a la dimensionalidad metodológica de toda la investigación. Esta metodología se deriva de problematizar la pregunta que consiste en señalar: ¿cuál es el discurso que el medio de comunicación La Nación construyó para sus noticias y editoriales, de mayo de 2007 hasta abril de 2008, sobre la educación pública costarricense?
En términos esquemáticos, se analizó el discurso que difundió el medio de comunicación La Nación en las noticias y editoriales sobre la educación pública costarricense de mayo de 2007 hasta abril de 2008. Esto se hizo a partir de profundizar en: a) tendencias temáticas en torno a la publicación de noticias y editoriales sobre educación pública costarricense en el periódico La Nación; b) clasificación y distinción de la forma en que se usan los elementos discursivos y retóricos que componen las noticias y editoriales del periódico La Nación, en torno a la educación pública en sus distintos componentes y c) un análisis de los marcos o esquemas (frames) discursivos que se reproducen en las noticias y editoriales acerca de la educación pública costarricense.

La investigación tuvo fines explicativos, así como una conjugación de datos cuantitativos como cualitativos, una triangulación metodológica que por lo demás, no fue sencilla $y$ sigue presentando consideraciones problemáticas para esta, como para otras investigaciones. La investigación contiene un total de 12 meses (mayo 2007-abril 2008) y se usaron técnicas de análisis de contenido y discurso. El análisis de contenido se refiere a recuperación de datos referidos a evidencia cuantitativa (cfr. Krippendorff, 1997), mientras que el análisis de discurso, como se señaló, se centra en el estudio del framing. En este sentido se debe tener presente que:

El framing está pensado para lograr extraer un espacio general de comprehesión, lo cual no da un acercamiento particular a los detalles $y$ aspectos secundarios. Lo que el framing pretende es establecer el mensaje central de una o una serie de documentación de algún tipo, especialmente al hablar de series de documentos. En esta investigación el framing propicia una articulación en torno a la centralidad de un discurso sobre el sistema educativo público del país, sin embargo temas alusivos a la educación pública referenciados en menos oportunidades, pueden perderse en el proceso de desarrollo de esta técnica (Carballo, 2009: 73). 
En aras de poder crear una lista de noticias a las cuales analizar y sistematizar en términos de signos, símbolos, significados, etc. que den pie a la reconstrucción de los frames, se empleó la ponderación cuantitativa de pesos por meses. Por medio de ella, se detectó saturación y liviandad en términos de la presencia mediática del tema del sistema educativo público, así como la combinación con otros temas como tecnología, criminalidad, etc. El siguiente cuadro 1, base para poder desplegar una selección proporcional de las noticias por meses para el estudio, tiene como referente de peso el cuadro 3 (ver más adelante) donde se decantan las cantidades por mes. En este cuadro 1 se evidencia la proporción de noticias que se toman para reconstruir los frames sobre protagonistas específicos: profesores, estudiantes y la institución (MEP).

\section{CUADRO 1}

DIVISIÓN DE LAS NOTICIAS

Y EDITORIALES SEGÚN MESES

\begin{tabular}{lcc}
\hline & NOTICIAS & EDITORIALES \\
\hline Mayo & 3 & 1 \\
Junio & 3 & 1 \\
Julio & 3 & 1 \\
Agosto & 3 & 1 \\
Setiembre & 3 & 2 \\
Octubre & 3 & 3 \\
Noviembre & 3 & $0^{*}$ \\
Diciembre & 4 & 1 \\
Enero & 3 & 1 \\
Febrero & 4 & 1 \\
Marzo & 3 & $0^{*}$ \\
Abril & 5 & 3 \\
\hline TOTAL & 40 & 15 \\
\hline
\end{tabular}

* No se registró ningún editorial sobre educación.

\section{CONTEXTO: LA EDUCACIÓN PÚBLICA COMO PREOCUPACIÓN DE ESTADO}

La educación pública ha tenido un recorrido fundamental a la par de la configuración del Estado Nacional Costarricense. Pese a que Costa Rica fue la provincia más alejada de la Capitanía General de Guatemala y se mostraba como una de las más atrasadas, es durante el desarrollo de la segunda mitad del siglo XIX y todo el siglo Xx, que la condición de la calidad educativa desplaza a las restantes naciones vecinas. Diferentes elementos explican esta condición. Elementos propios de cada país, como guerras $y$ dictaduras atroces que no permitieron lo que en Costa Rica se logró generar, sin olvidar las contradicciones propias que en el país se gestaban. Sin extenderse en períodos históricos o en elementos estadísticos, vale la pena resaltar algunos puntos que hacen relevante y visible el estudiar el peso socio-económico y socio-político que ha tenido el sistema educativo, así como repensarlo en términos discursivos.

El siglo XIX en Costa Rica, acabó con una serie de instituciones fragmentadas que permitieron regenerar otro tipo de instituciones, que a la larga terminan por ser las que hoy vivenciamos. El cierre de la Universidad de Santo Tomás, antes casa de Estudios de Santo Tomás, fue relativo, ya que muchas unidades de estudios como agricultura y derecho, entre otras pocas, siguieron operando hasta la conformación o reconformación de un centro de Estudios superiores como lo es la Universidad de Costa Rica. Además, la paulatina elaboración Estatal decanta en la creación del Ministerio de Educación, ya avanzado el siglo xx, lo que orienta, tanto una visión de centralidad Estatal, así como responsabilidades ciudadanas en torno a brindar educación a la población, situación que se refleja y se consolida en la misma constitución costarricense de 1949. Se puede señalar que del siglo XIX, se tiene:

... [o] se promueve una lógica de fomento nacional, un proceso civilizatorio $y$ modernizante. Es decir que los procesos de educación en general de las y los costarricenses estuvieron íntimamente unidos con el proceso de identificación cívica $y$ al mismo tiempo con el fomento de una visión de país, que tenía como ejemplo inmediato repúblicas europeas como fue el caso de España, Francia e Inglaterra (Carballo, 2009: 84).

En el siglo Xx, se profundiza en un modelo más condensado institucionalmente, así: 
El contexto en que se fue concretando todo el aparato estatal que regía la educación formal estuvo inserto en una serie de polémicas en torno a los "intereses nacionales" y la formación de ciudadanas y ciudadanos, especialmente estos últimos, con capacidades útiles y habilidades de convivencia. El resultado de este debate social y político fue que se gestó todo una articulación en donde la continuidad en el sistema educativo obtuvo rasgos unificadores y así se prosiguió con una institucionalización del modelo de educación costarricense (Carballo, 2009: 90).

Se remarcan elementos generales de lo que se ha vislumbrado como la consolidación del Estado en relación con la educación pública. Sin embargo, es importante señalar algunos otros elementos que colaboren con el marco de análisis histórico que permita entrever la importancia y el papel que la educación pública tiene en diferentes niveles: político, económico, discursivo, nacionalista, entre otros posibles. A finales del siglo $\mathrm{xx}$, otras visiones empiezan a ser más polemizadas en el análisis de la educación pública a nivel nacional como a nivel internacional. La privatización, la vinculación con los Programas de Ajuste Estructural, así como, las modalidades de reformas educativas (Solano, 2001; Gutierrez y Jiménez, 2000 y Popkewitz, 1997) son elementos críticos con los que se cierra el siglo XX. Además, las problemáticas de cobertura y de calidad empiezan a avizorarse con más preocupación, así lo resaltan los estudios del Estado de La Nación (cfr. 2006; 2005a; 2005b), así como el Estado de la Educación (cfr. 2008a; 2008b). Estos informes señalan problemas a nivel dicotómico, problemas de cobertura y calidad a nivel de lo rural $y$ lo urbano, así como diferencias de género, entre otras. Pero se coincide con que el desarrollo de la educación se ha estancado en ciertos rubros, lo cual modela una postura para cambiar o variar el tipo de educación que se imparte. Este es un punto importante porque redunda en la gama de propuestas que pasan por filtros ideológicos y discursivos como el que se hace en esta reflexión sobre un medio de comunicación.

\section{LA IMPORTANCIA DEL MANEJO DE DATOS CUANTITATIVOS}

Uno de los elementos que permite entrever tendencias es la recuperación de datos cuantitativos que nos den cierta idea de regularidad. En este sentido, se puede visualizar una serie de datos que permiten en primera instancia, describir un escenario de presencia mediática del tema en estudio: el sistema de educación pública. También, se pueden constatar ciertas irregularidades que dan paso al análisis, comparando lo regular con lo irregular.

El estudio consiste en un análisis de 12 meses, iniciando en mayo de 2007 y concluyendo en abril de 2008. El cuadro 2 muestra la distribución por pesos de las noticias y otras informaciones que fueron recuperadas para hacer el estudio.

CUADRO 2

TOTAL DE NOTICIAS E INFORMACIONES EN GENERAL SOBRE EDUCACIÓN PÚBLICA EN EL PERIÓDICO LA NACIÓN. MAYO 2007 - ABRIL 2008

\begin{tabular}{lcc}
\hline INFORMACIONES & FRECUENCIA & PORCENTAJE \\
\hline Otros* $^{*}$ & 42 & 12,3 \\
$\diamond$ Editoriales & 24 & 7 \\
$\diamond$ Campos pagados & 18 & 5,3 \\
Noticias & 300 & 87,7 \\
\hline TOTAL & 342 & 100 \\
\hline
\end{tabular}

* Otros: Está conformado por editoriales y campos pagados de agrupaciones sindicales y del Ministerio de Educación (no se incorporan los avisos, campos pagados o anuncios de las universidades públicas ni las privadas).

Fuente: elaboración propia con datos de La Nación.

Lo primero importante de señalar, en términos de la aparición mediática, es la comparación entre los meses de un año y el otro. Lo relevante de esta comparación es mostrar la equiparación de pesos entre los años, pese a que el 2007 tiene el doble de meses estudiados que el 2008. En el gráfico 1 se observa la casi nula diferencia en pesos. 


\section{GRÁFICO 1}

AÑO EN QUE LAS NOTICIAS SE PUBLICARON 2007-2008

EN PORCENTAJES

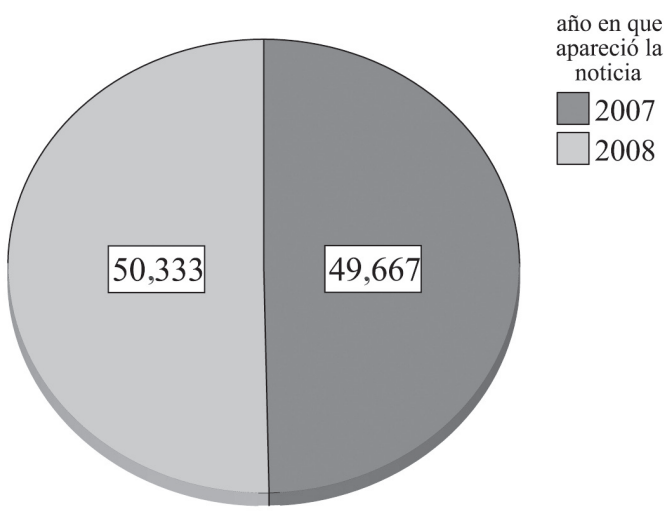

Fuente: elaboración propia con datos de La Nación.

A partir de especificar el año según pesos, también se puede especificar los meses según sus cantidades, ya que esto demuestra centralidad o concentración, la cual puede estudiarse temáticamente, es decir, precisar el tipo de información según temáticas que se abordaron. En el caso de todas las informaciones recuperadas: noticias, editoriales y campos pagados, los pesos no varían casi en absoluto. Esto revela una concentración de información o noticias en el año 2008, lo cual induce a estudiar con más precisión ciertos meses, dentro de los cuales se puede adelantar el mes de abril.

El cuadro 3 muestra una distribución de cantidades de noticias según meses estudiados, lo cual evidencia la concentración de manera específica.

La cantidad igual de noticias de 2008 frente al 2007, a pesar de la diferencia de cantidad de meses, se puede postular, desde un punto de vista hipotético $y$ de trabajo, de la siguiente manera:

... dos explicaciones surgen para ayudarnos a entender el por qué de una cantidad de noticias en el año 2008 (4 meses) similar a la cantidad de noticias del 2007 (8 meses). En primer lugar debemos recordar (...) la entrada a clases. Y en segundo lugar recordar el acontecimiento de la huelga de educadores que se llevó a cabo en el mes de abril 2008 (Carballo, 2009: 121). [Sin embargo, ambas hipótesis solo explican de manera reducida el fenómeno].

\section{CUADRO 3}

PUBLICACIONES DEL DIARIO LA NACIÓN RESPECTO A LA EDUCACIÓN PÚBLICA COSTARRICENSE DIVIDIDO SEGÚN EDITORIALES Y CAMPOS PAGADOS POR MES Y AÑO

\section{(VALORES ABSOLUTOS Y PORCENTAJES)}

\begin{tabular}{lccc|lccc}
\hline & $\begin{array}{l}\text { SOLO NOTICIAS RECOPILADAS } \\
\text { SIN EDITORIALES NI CAMPOS PAGADOS }\end{array}$ & \multicolumn{3}{c}{$\begin{array}{c}\text { TOTAL DE INFORMACIÓN RECOPILADA } \\
\text { CON EDITORIALES Y CAMPOS PAGADOS }\end{array}$} \\
\hline MES & AÑ & FRECUE. & $\%$ & MES & AÑO & FRECUE. & $\%$ \\
\hline Abril & 2008 & 71 & 23,6 & Abril & 2008 & 81 & 23,7 \\
Febrero & 2008 & 32 & 10,6 & Febrero & 2008 & 36 & 10,5 \\
Diciembre & 2007 & 27 & 9 & Diciembre & 2007 & 30 & 8,8 \\
Marzo & 2008 & 27 & 9 & Marzo & 2008 & 27 & 7,9 \\
Noviembre & 2007 & 24 & 8 & Noviembre & 2007 & 25 & 7,3 \\
Agosto & 2007 & 20 & 6,6 & Mayo & 2007 & 25 & 7,3 \\
Enero & 2008 & 21 & 7 & Agosto & 2007 & 24 & 7,0 \\
Junio & 2007 & 19 & 6,3 & Junio & 2007 & 23 & 6,7 \\
Mayo & 2007 & 19 & 6,3 & Enero & 2008 & 22 & 6,4 \\
Octubre & 2007 & 16 & 5,3 & Octubre & 2007 & 20 & 5,8 \\
Julio & 2007 & 12 & 4 & Julio & 2007 & 15 & 4,4 \\
Setiembre & 2007 & 12 & 4 & Setiembre & 2007 & 14 & 4,1 \\
\hline TOTAL & 300 & 100 & TOTAL & & 342 & 100 \\
\hline
\end{tabular}

Fuente: elaboración propia con datos de La Nación. 
En primer lugar, hablar de la entrada a clases redunda no solo en lo que se podría llamar más noticias, sino en un tipo determinado de noticias. En este sentido, explica cantidad pero no necesariamente lo cuantitativo denota el contenido temático. Es aquí donde es impor- tante interpretar los datos recuperando el tipo de contenido (tabla 1). Si se toma el mes de febrero por ejemplo, se puede ver lo señalado, además se pueden especificar noticias e incluso, se fortalecería aún más el análisis si conjugamos temas por tamaños.

TABLA 1

LISTADO DE LOS TITULARES DE NOTICIAS REGISTRADOS IGUALES O MAYORES A 1 PÁGINA

FEBRERO, 2008

\begin{tabular}{lc}
\hline TITULAR & FECHA \\
\hline Engorrosos $^{*}$ trámites impiden mejorar infraestructura escolar & 2 \\
Pruebas no pueden ser autopsias & 2 \\
Maestros incapacitados en febrero ganan más que quienes dan clases & 5 \\
INA se declara incapaz de formar técnicos para empresas & 6 \\
MEP replantea enseñanza en los colegios técnicos & 11 \\
Curso lectivo comienza con faltante de 700 educadores $^{(2)}$ & 12 \\
\hline
\end{tabular}

(1) Las noticias se encuentran entre el rango de 1 a 1 y 3/4 de página. Solo una es superior a dicho rango.

(2) Noticia de 2 páginas.

* Las palabras en cursiva son para resaltar calificativos.

Fuente: elaboración propia con datos de La Nación.

La entrada a clases explica la aparición de más noticias, ya que dentro de la teoría de la noticiabilidad y de la interpretación que el medio haga sobre un fenómeno - considerado social y políticamente importante $y$ determinante- este momento marca pautas de conducta de amplia diversidad social en el país. Lo relevante del estudio de regularidad es mostrar que estas noticias no solo incrementan, sino que incrementa una forma de presentarse, según una postura ideológica que se denota discursivamente.

En el caso de la entrada a clases del medio se señala que:

... el hecho que aumente la cobertura sobre educación pública durante la entrada de clases no quiere decir que se aborden temas relativos a dicho fenómeno, el de la entrada de clases. Es decir, $L a$ Nación en el mes de febrero no enfatizó, aunque sí hizo mención, sobre los temas de becas, acceso efectivo que se hizo a materiales por parte de los estudiantes, nombramientos, causas de deserción, entre otros fenómenos, pero sí se concentró en los recursos que sí faltaban, tanto en el área docente como en el área de los materiales, lo cual lo ampliaron según el tamaño de las noticias y la perspectiva del titular. Existió un énfasis respecto a la estructura física de la noticia (tamaño específicamente), donde unos temas se enfocaron en las tamaños más grandes -fallas - y otros en los más pequeños - aportes o desarrollo (Carballo, 2009: 125-126).

Partiendo de enjuiciar no la cantidad, sino la relación que la cantidad tiene con lo que está presente en materia de contenido, se puede hacer una serie de relaciones para mostrar por medio de las regularidades y las tendencias, los posicionamientos ideológicos. Sin profundizar en muchos de estos elementos, se puede tomar un tema específico con qué ilustrar el uso del recurso cuantitativo para profundizar el aspecto discursivo sobre la forma de construir la comprensión sobre la educación pública.

El caso del mes de abril, su estudio más profundo, adentra en una reflexión importante en términos de cómo entender el resto del conjunto de información que hasta el momento se tenía. Abril fue el mes que más información -71 noticias, $23 \%$ del total de noticias- contabilizó, inclusive se detectó que tiene el doble de noticias que el segundo mes con mayor cantidad (febrero). 
GRÁFICO 2

RELACIÓN DE LAS NOTICIAS RECOPILADAS

CON LA HUELGA DE ABRIL DE 2008

EN PORCENTAJE

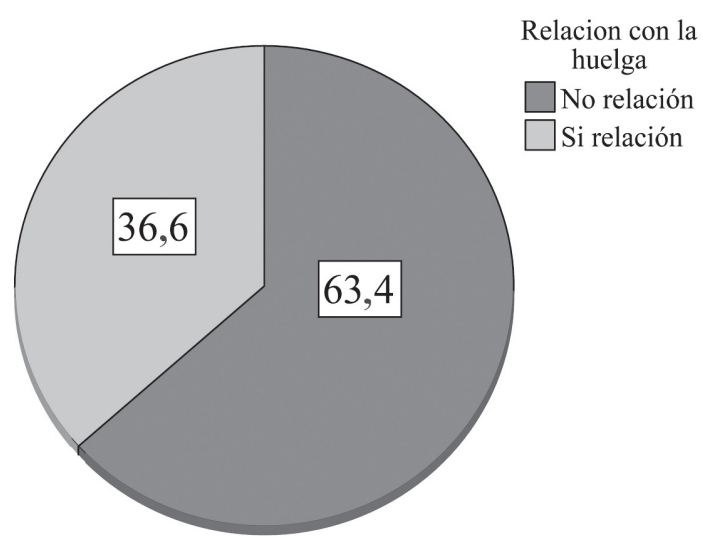

Fuente: elaboración propia con datos de La Nación.

Además, como lo indica el gráfico 2, las noticias sobre la huelga no llegan ni al 40\% de todas las noticias sobre educación pública. Solo como reflexión crítica valdría la pena preguntarse si un evento distinto, deportivo tal vez, tendría más o menos un $40 \%$ en términos de la concentración mediática.

Una de las hipótesis de trabajo fue la valoración del fenómeno de la huelga que ocurrió en abril de 2008. La huelga explica de alguna manera la cantidad de noticias, pero cuando se es más minucioso se puede ver que la huelga tuvo pesos marginales en tamaño y aparición, así como la presencia de noticias promocionadas en la portada del mismo diario (cfr. Carballo, 2009).

Las noticias sobre la huelga no fueron las más grandes, ni las más publicitadas en la portada. La siguiente cita ilustra lo señalado:

Dos puntos importantes de rescatar son en primer lugar que La Nación sí evidenció en sus portadas la huelga que acontecía. Pero también se debe tener en perspectiva la proporcionalidad de estas noticias anunciadas en la portada a partir del total de días (portadas) que estaban a disposición. Es decir, de 30 días que conforman el mes de abril, solo 6 portadas $(20 \%)$ contaron con algún contenido de la huelga (...). Además encontramos que de esas 6 noticias ${ }^{5}$ solo una fue central, por lo que la cobertura en términos de anunciación en portada fue relativamente baja, no solo en términos de cantidad de portadas disponibles, sino también en términos de cantidad de noticias que el periódico publicó sobre la huelga. Es decir, de 25 noticias sobre la manifestación de profesores, solo $7(28 \%)$ se enunciaron en portada, $y$ principalmente de manera secundaria (Carballo, 2009: 142).

En resumen, se puede argumentar que el diario no dejó de visualizar esta manifestación social específica, la huelga, pero sí utilizó información relativa a otros aspectos de la educación pública para opacar, sin dejar de publicar información sobre la huelga, lo cual nos hace pensar en una postura, tanto ideológica como consciente.

El tema de la huelga fue retomado en $L a$ Nación, sin embargo las noticias tendieron a ser de las más pequeñas (un número muy bajo de noticias de gran tamaño). El 36\% del total de noticia publicadas en abril del 2008 fue referente a la huelga de educadores, sin embargo el $64 \%$ de las noticias restantes abordaron temáticas distintas y con más espacio proporcional que las noticias sobre la huelga. Es decir, la huelga fue abordada por el diario continuamente, más fue opacada por las restantes noticias sobre educación pública (sin olvidar las noticias que no tratan sobre educación pública), lo que minimizó el impacto mediático que se le pudo haber dado (Carballo, 2009: 145).

Este ejemplo específico busca evidenciar el potencial analítico que la interpretación puede proporcionar aunada con una honesta

$5 \quad$ Recordemos que dos noticias que aparecieron en portada fueron publicadas el mismo día, viernes 25. Por lo que, si se cuenta la noticia del día 15 de abril, el total de noticias en portada se reduce a seis por encontrarse la reiteración en una portada. 
y rigurosa recolección de datos cuantitativos. No solo regularidad, sino también aporta posibilidades para profundizar en los análisis. Eso depende de quienes preparan la metodología, la tabulación, así como el resultado de este proceso, todo conectado, hablando entre sí, pero sin olvidar el grado de complicación que puede surgir en su elaboración.

\section{EL DISCURSO DESDE EL ABORDAJE FRAMING}

Recordando los elementos que se esgrimieron en torno a la metodología y técnica del framing, este acápite versa sobre entrever la reconstrucción que se hizo sobre tres protagonistas específicos que forman parte del sistema educativo público: los profesores, los estudiantes y la institución (MEP). Esta reconstrucción se basa en las noticias recuperadas en los meses estudiados. Desde el punto de vista metodológico, se suprimen los cuadros y sistematización que se llevó a cabo para dar con las siguientes reflexiones y resultados, tendiendo a ciertas consideraciones amplias; sin embargo, para tener un despliegue completo de listados de referencias específicas se puede revisar el trabajo base en: Carballo, 2009, donde se suministra tanto las tablas de sistematización, los cuadros y tablas por protagonista y temática, así como, cada una de las hojas de codificación de cada noticia y editorial ${ }^{6}$ incorporada al estudio.

\subsection{PROTAGONISTAS: LAS Y LOS PROFESORES}

La reconstrucción desde los frames indica que el o la profesora se presenta con una serie de elementos simbólicos que redundan en que está desactualizado y que se vuelve un revoltoso incitador o un "rebelde", cuando estimula o realiza acciones más allá del aula o por fuera de su institución educativa. Estas actividades no son absolutas, es decir, las actividades con alta tendencia política como huelgas o enfrentamientos críticos con lo políticamente correcto, son recriminados y establecidos como

$6 \quad$ En este artículo solo se procedió a desarrollar un análisis a partir de las noticias, para ver el estudio específico de los editoriales y campos pagados se puede consultar: Carballo, 2009. no deseables en el medio, estos son el tipo de sanciones político-comunicativas que realiza el medio cuando las y los docentes ejercen niveles de ciudadanía, aludiendo a su vez a su condición de profesores. En Noticias 1, se muestra una pequeña ejemplificación de calificativos que redundan en esta dinámica discursiva sobre las y los docentes.

$\frac{\text { NOTICIAS } 1}{\text { 1. Hasta que profesores no regresen a las aulas (abril 2008). }}$
2. Reciben mala información de APSE (abril 2008).

Fuente: elaboración propia.

Además, existe una ambigüedad y ausencia de reflexión sobre problemáticas de los profesores, por un lado, se les dice sacrificados y ejemplo (cfr. Carballo, 2009: 202), pero por otro, cuando se toman acciones para superar el sacrificio y pasar al plano de los derechos y las libertades de expresión, como por ejemplo, una huelga, el medio reacciona en el sentido de mantener orden $y$ estabilidad social, pese a que el mismo medio en sus acciones discursivas está generando conflicto $y$ choque simbólico $y$ discursivo. En términos generales, se da con esta dicotomía analítica:

... el profesor puede ser víctima cuando está dentro de la estructura, y el discurso institucional de la lógica del Estado. Es decir, cuando trabaja dentro del Estado o de la estructura estatal, el discurso de La Nación es a señalarlo como una víctima más del sistema. [...] Cuando no siguen lo encomendado social e institucionalmente, $y$ presentan una confrontación al sistema, se les encasilla y se confunden. Es decir, por un lado se les tilda de incompetentes o ignorantes por ser engañados, por dirigentes sindicales, $y$ por otro se les califica como vagos o incapaces (Carballo, 2009: 204).

Aunque se puede desarrollar más puntos (ver: Carballo, 2009) los elementos de desactualización, rebeldía y contradicciones en la misma construcción de los argumentos de noticia a 
noticia, entrevén que el educador se aproxima más a un instructor que a una persona con quien compartir conocimientos, sino un transmisor en su sentido más técnico. Pero que no olvida elementos semánticos de la romántica condición con que el docente ha sido construido en la historia política del país, la visión del sacrificio y la educación como fundamento de la superioridad del país en términos de región. Un último elemento importante que vincula la reflexión cuantitativa con la cualitativa, es hacer énfasis en que las noticias sobre tecnología, futuro $y$ desarrollo, se dieron en los meses de entrada a clases, así como en abril -el mes de la huelga - (cfr. Carballo, 2009: 213). Esto puede servir como estímulo para pensar que frente a una posición política contraria a posturas ideológicas, tanto de Estado como privadas, la visión de mercado y de competitividad/ individualidad ejercen un papel protagónico en la contienda por influir en el sistema educativo, desde el campo tanto mediático, como en otros. Es decir, en un punto específico, la cantidad de noticias sobre educación pública es alta $y$ la influencia sobre esas noticias versa sobre proponer las temáticas y sus modalidades de comprensión en el sistema en general -frame.

\subsection{PROTAGONISTAS: LAS Y LOS ESTUDIANTES}

En una línea similar con las y los estudiantes se operó una dicotomía explicativa de cómo tratar a estos protagonistas. O como el prototípico, el modelo a seguir, en otras palabras estudiante con futuro o sin futuro, que en su caso sería el criminal o criminal en potencia.

Es decir, el frame que se contrapone al estudiante no prometedor, es aquel que se le da visión global, $y$ herramientas globales. Por tanto, es una apuesta simbólica, donde se procede a mostrar un modelo de sociedad mundial, así como las características de las personas que dicho modelo de sociedad permite. Además surge también el estudiante prototípico cuando es este quien rescata el orden social, cuando se genera una ruptura del orden, este estudiante defiende el orden, y con su acción rescata la "esperanza" de volver a la senda del camino correcto (Carballo, 2009: 215-216).

Estos elementos condensan una gran cantidad de información que se reconstruye a partir de incorporar elementos o temáticas con que se conjugaron las noticias sobre la educación, temáticas como el inglés, la tecnología, colegios científicos, las drogas, entre otras. En el caso de la apelación de futuro, lo científico se superpone a otras visiones positivas dentro del sistema educativo, en Noticias 2 vemos un ejemplo de lo que se especifica sobre los colegios científicos frente a otras modalidades educativas.

\section{NOTICIAS 2}

1. Sus estudiantes destacan (marzo 2008)

2. Desarrollan más habilidades (marzo 2008)

3. Dotar a los estudiantes de un laboratorio (abril 2008)

Fuente: elaboración propia.

El tema de la globalidad es un importante insumo analítico. Sin despreciar el elemento de la importancia comunicativa y de acceso a herramientas de diverso tipo en aras de igualdad, la confrontación entre quienes se presume, tienen futuro $y$ quienes no, no necesariamente estimulan a vislumbrar un futuro colectivo, sino que está expuesto como resultado de una serie específica de consignas, que más importante que un estímulo de socialidad o reciprocidad, están basadas en escalar individualmente, así como, de innovación técnica. Las propuestas de la mayoría de las noticias no son necesariamente democratizar o asegurar derechos, ideas que en cierto sentido, estimulan de alguna manera la disminución de la desigualdad o la mejoría de los que no están tan bien, sino que estas noticias lo que propician es velar por entrar en los "senderos" de la globalización, sin necesariamente proponer mecanismos, razones de estudios o alguna evidencia de proceso, sino que, es más apelando a las consignas ya presentes en la discusión y debate público acerca de cómo operar socio-económicamente.

Cuando nos referimos al mirar hacia afuera, estamos resignificando la metáfora 
aparentemente escondida dentro del mensaje del discurso. Son los que aspiran a más o los que ven, los que tienen visión. Es un juego que combina los usos retóricos del lenguaje con un entramado simbólico del contexto de vida y propuesta de vida. La globalización que tiene un matiz de noción internacional postula que la persona en formación también debe estar globalizada e internacionalizada. Entonces no es meramente un programa o proyecto de conocimientos, sino un proyecto socio-político que avala un modelo de vida, según pautas de globalización, $y$ de mercado libre, que es la consigna que se señala a la hora de hablar de apertura $y$ globalización (Carballo, 2009: 211).

El "buen" estudiante, el modelo de estudiante, está cerrado simbólicamente en una lista mínima de elementos, entre ellos, técnico o en su ampliación tecnologizado e igual de importante a-político. Es decir, no manifestando acciones, más allá de cuidar el ambiente, limpiar una playa o participar en actividades comunales/escolares. Pero no reconociendo que el y la estudiante son al mismo tiempo, actores importantes de muchas comunidades, esta faceta es suprimida en orden a su aplicación académica.

\subsection{PROTAGONISTAS: INSTITUCIÓN (MEP)}

El Ministerio de Educación actúa bien o mal, según ejerza e imponga el orden y las condiciones tecnológicas e infraestructurales según el estándar global. Por un lado, La Nación critica al Ministerio cuando no cumple con elementos específicos de calidad, pero cuando las personas, las y los ciudadanos, docentes y estudiantes, reclaman abiertamente por la solución de dichos derechos, el Diario se ubica alejado y esgrime una postura de orden, control y jefatura.

El tratamiento de una eficacia en el manejo del ministerio respecto a los otros meses hace pensar en que el hecho de la huelga estimula una organización estratégica del medio La Nación, lo cual propone noticias que realcen el aporte del MEP (a diferencia de otras ocasiones) para, una vez más, deslegitimar el movimiento de profesores, es decir la huelga. De las 40 noticias seleccionadas vemos como la tendencia acerca de las acciones afirmativas $y$ toma de decisiones del MEP se concentran en los meses de febrero, marzo y abril del 2008. En cambio las noticias que muestran un estado defectuoso del MEP son más constantes a lo largo del período de estudio (Carballo, 2009: 221).

El MEP tiene responsabilidades morales, legales, constitucionales, etc. Sin embargo, no queda siempre claro cual es la conexión entre necesidad de las personas, el país y el modelo y el tipo de educación que se debe impartir. En este sentido:

... es una institución con órdenes, jerárquicos o cadenas de mando. Por tanto tiene una responsabilidad política legitimada. Este es el principal contacto, conexión o símbolo que retoma constantemente La Nación cuando simpatiza con alguna idea o existe un tercer factor ideológicamente contrario a ambos [...] Es decir, el control sobre las movilizaciones sociales se buscan contener con estrategias políticas directas o indirectas (conscientes o inconscientes) de entes sociales (Carballo, 2009: 225).

El siguiente cuadro de Noticias 3 concentra dos de las principales y recurrentes formas de presentación de autoridad.

\section{NOTICIAS 3}

1. Retendrá salarios (abril 2008).

2. Pidió lista de los que participan en huelga (abril 2008).

Fuente: elaboración propia.

En términos generales, La Nación usa y expresa las facetas más jerárquicas de la institución (cfr. Carballo, 2009: 224), mientras que también sanciona las condiciones de falencias de 
dicha institución, claro, apelando a un modelo no necesariamente amplio de lo que el sistema educativo debería por derecho proporcionar a la población.

\section{CONCLUSIONES}

Muy concretamente se puede señalar que en términos del análisis de las noticias - no incluyendo los editoriales, ni otras formas de información- se puede hablar de ideas de educación eminentemente tecnificada, de pragmática educativa y la amenaza de la desorganización educativa, como frames "necesarios" para comprender el fenómeno de la educación pública. "Poner orden en la casa" (La Nación citada en Carballo, 2009: 247), es la apelación del Diario, pero lo importante es el tipo de ordenamiento que se estimula, se propone y se impulsa. Como idea general, relevante en términos públicos se puede rescatar que:

En la misma línea, la delimitación simbólica de los que utilizan la educación pública - estudiantes pobres- estructura un esquema de análisis para señalar un tipo asistencial de educación, que el diario construye en sus noticias y editoriales, propio de un "mundo estatal" que es, según el periódico, inoperante e inconsistente con el sistema socio-económico actual (Carballo, 2009: 253-254).

Otra idea igualmente importante, es revelar la contradicción que surge en este análisis sobre el medio; una contradicción argumentativa, pero no necesariamente propia del tratamiento ideológico de La Nación.

Las víctimas vienen a ser los estudiantes que pierden clases porque los docentes no asisten, pero no se pone a los docentes como víctimas que sacrifican su tiempo $y$ responsabilidades para ir a protestar por sus intereses $y$ las de sus familias, es decir, existe un control de lo simbólico para usarlo como un frame para encasillar quienes son $y$ pueden ser víctimas $y$ quienes no son víctimas. Igual, el héroe es aquel o aquella docente que se queda en el aula y "sufre" su destino valientemente, el sacrificio como mito del docente; $y$ el estudiante ya no es víctima cuando fuma droga y le hace la vida difícil al docente, sino un producto de las malas juntas, de un aparato estatal deficiente, por lugar de procedencia, pero pocas veces como producto de la combinación de factores claramente delimitados $y$ analizados (Carballo, 2009: 255).

La idea es contradictoria porque los que reclaman por lo que supuestamente el medio también reclama, es decir, por mejoras, son vilipendiados por La Nación por el mismo acto. El hecho es claro, tanto por reclamar como por la forma en que lo hacen, así como, por su matiz ideológico, es decir, la huelga y las razones que motivan a hacerla: derechos salariales, mejoras en atención y algo que La Nación nunca incorpora como una palabra clave, el respeto a la labor y al trabajo. A diferencia de la cantidad de veces que la tecnología y el inglés es publicada, el respeto no es incorporado. La reconstrucción mediática, el discurso sobre el sistema educativo público del medio es espuria, inconsistente con una propuesta amplia de derechos y esto es importante de resaltar. El acceso a medios tecnológicos y de otro tipo es parte de la consigna de derechos y de igualdad, pero la labor educativa versa sobre elementos de socialidad, respeto, arte, etc. Sintetizar la educación en elementos de rigor competitivo, así como, de orientación global, no necesariamente impulsan un ímpetu colectivo, ni escenarios de derechos, así como, deja una inconsistente visión de individualidad, que para nada debe descartarse y que resulta fundamental para la educación de las personas y en la reproducción de nuestros discursos.

\section{FUENTES DE INFORMACIÓN}

Alsina, Miquel R. La construcción de la noticia. $2^{\circ}$ Edición. Barcelona, España: PAIDos Comunicación, 1993.

Araya, Alexander. "Estudio sobre la construcción de las representaciones sociales sobre la inseguridad ciudadana en la prensa escrita costarricense: el periódico La Nación 2003-2005". 
[Tesis de Licenciatura en Sociología]. Universidad de Costa Rica, 2008.

Arias, Cristina y Bermúdez, Mario. "Alta tensión en el ICE: la corriente de la privatización. Política informativa y editorial del periódico La Nación 1987-2004". [Tesis para optar por la Licenciatura en Ciencias de la Comunicación Colectiva]. Universidad de Costa Rica, 2006.

Ariño, Antonio. "Ideologías, discursos y dominación". [Artículo digital]. REIS, 1997: 197-219.

Beirute, Tatiana. "Las representaciones sociales sobre la violencia intrafamiliar contra las mujeres en la prensa costarricense: un estudio sociológico del periódico La Nación y el Diario La Extra en el año 2005". [Tesis de Licenciatura en Sociología]. Universidad de Costa Rica, 2006.

Carballo, Pablo. "El discurso mediático sobre el sistema educativo público costarricense. Estudio y análisis ideológico/discursivo del diario La Nación. Mayo del 2008Abril del 2009". [Tesis de Licenciatura en Sociología]. Universidad de Costa Rica, 2009.

Foucault, Michel. Arqueología del saber. $22^{\circ}$ edición. México: Siglo XXI editores SA. 2006

Foucault, Michel. Un dialogo sobre el poder. $2^{\circ}$ impresión. Madrid, España: Alianza Materiales, 1984.

Jiménez Ardón, Oscar. "La prensa y la ideología dominante en Costa Rica. Análisis sociológico del discurso religioso del periódico La Nación (1979-1987)". [Tesis de Magíster Scientiae en Sociología]. Universidad de Costa Rica, 1989.

Krippendorff, Klaus. Metodología de análisis de contenido. Teoría y práctica. $1^{\circ}$ reimpresión. Barcelona, España: PAIDós Comunicación, 1997.

López, Olimpia. Sociología de la educación. $8^{\circ}$ reimpresión. San José, Costa Rica: EUNED, 2004.

Mannheim, Karl. Ideología y Utopía. México: Fondo de Cultura Económica, 2004.
Martín Cañas, Kattia. "El combo del ICE y La Nación: análisis para un periodismo socialmente responsable". [Tesis Magíster Scientiae en Comunicación]. Universidad de Costa Rica, 2004.

Oconnor, D.J. Introducción a la filosofía de la educación. Buenos Aires, Argentina: Editorial Paidós, 1971.

Popkewitz, Thomas S. Sociología política de las reformas educativas: el poder-saber en la enseñanza, la formación del profesorado y la investigación. $2^{\circ}$ edición. Madrid: Ediciones Morata, 1997.

Programa Estado de la Nación en Desarrollo Humano Sostenible. Estado de la Educación Costarricense 2. San José, Costa Rica, 2008a.

Programa Estado de la Nación en Desarrollo Humano Sostenible. Estado de la Región en Desarrollo Humano Sostenible. San José, Costa Rica, 2008b.

Programa Estado de la Nación en Desarrollo Humano Sostenible. Duodécimo Informe sobre el Estado de la Nación en Desarrollo Humano Sostenible. San José, Costa Rica, 2006.

Programa Estado de la Nación en Desarrollo Humano Sostenible. Estado de la Educación Costarricense. San José, Costa Rica, 2005a.

Programa Estado de la Nación en Desarrollo Humano Sostenible. Undécimo Informe sobre el Estado de la Nación en Desarrollo Humano Sostenible. San José, Costa Rica, 2005b.

Ramírez, Mario y Pereira, Denia. "Las reglas del juego: la censura y el derecho informativo en el habitus de los periodistas costarricenses". [Tesis interdisciplinaria de Licenciantura en Periodismo y Derecho].Universidad de Costa Rica, 2004.

Sandoval, Carlos y Al-Ghassani, Anwar. Inventario de los medios de comunicación de Costa Rica. San José, Costa Rica: Escuela de Ciencias de la Comunicación Colectiva UCR, 1990.

Valitutti Chavarria, Gina. "Ideología y poder. El discurso del periódico La Nación con 
respecto al proceso político nicaragüense (1979-1986)". [Tesis de Magíster Scientiae en Sociología]. Universidad de Costa Rica, 1992.

Trigueros, Joaquín. "Framing del Tratado de Libre Comercio (TLC) en la prensa escrita de opinión en Costa Rica durante el período del referendo". [Tesis de Magister Scientiae en Comunicación]. Universidad de Costa Rica, 2008.

Van Dijk, Teun A. "El estudio del discurso". En: Van Dijk, Teun A. (comp.). El discurso como estructura y proceso. Estudios sobre el discurso I. Una introducción multidisciplinaria. Volumen I. Gedisa Editorial, 2003: 21-65.
Van Dijk, Teun A. "El discurso como interacción en la sociedad". En: Van Dijk, Teun A. (comp.). El discurso como interacción social. Estudios sobre el discurso II. Una introducción multidisciplinaria. Volumen 2. Gedisa Editorial, 2000: 19-66.

Zeledón, Mario. La Desinformación de la prensa en Costa Rica: un grave peligro para la paz. Instituto Costarricense de Estudios Sociales. Heredia, Costa Rica, 1987.

Fechas de ingreso: 13/02/2011 Fechas de aprobación: 16/06/2011 